\title{
Avaliação da comunidade de macroinvertebrados aquáticos como ferramenta para o monitoramento de um reservatório na bacia do rio Pitangui, Paraná, Brasil
}

\author{
Ivana F. Barbola ${ }^{1}$, Marcos F. P. G. Moraes ${ }^{2}$, Tathiane M. Anazawa ${ }^{3}$, Elynton A. Nascimento ${ }^{4}$, \\ Everton R. Sepka ${ }^{5}$, Cleber M. Polegatto ${ }^{6}$, Julianne Milléo ${ }^{1} \&$ Guilherme S. Schühli $^{7}$
}

1. Departamento de Biologia Geral, Universidade Estadual de Ponta Grossa, Av. Carlos Cavalcanti, 4748, 84031-620 Ponta Grossa, PR, Brasil. (ibarbola@yahoo.com.br; jumilleo@gmail.com)

2. Coordenação do Curso de Tecnologia em Alimentos, Universidade Tecnológica Federal do Paraná, Av. Monteiro Lobato, s/n, km 04, 84016-210 Ponta Grossa, PR, Brasil. (marcospgm@yahoo.com.br)

3. Divisão de Processamento de Imagens, Instituto Nacional de Pesquisas Espaciais, Av. dos Astronautas, 1758, 12227-010 São José dos Campos, SP, Brasil. (tathimay@yahoo.com.br)

4. Departamento de Biologia, Pós-Graduação em Entomologia, Faculdade de Filosofia, Ciências e Letras de Ribeirão Preto, Universidade de São Paulo, Av. Bandeirantes, 3900, 14040-90 Ribeirão Preto, SP, Brasil. (elynton@yahoo.com)

5. Departamento de Química e Biologia, Universidade Tecnológica Federal do Paraná, Av. Sete de Setembro, 3165, 80203-901 Curitiba, PR, Brasil. (evertonsepka@yahoo.com.br)

6. Rua General Osório, 1362, 14010-100 Ribeirão Preto, SP, Brasil. (cleber.ras@gmail.com)

7. Empresa Brasileira de Pesquisa Agropecuária - Florestas, Estrada da Ribeira, km 111, Caixa Postal 319, 83411-000 Colombo, PR, Brasil. (schuhli@gmail.com)

\begin{abstract}
Evaluation of the aquatic macroinvertebrate community as a tool for monitoring a reservoir in the Pitangui river basin, Paraná, Brazil. Benthic and nektonic macroinvertebrates play an important role in the structure and function of aquatic ecosystems and their distribution is influenced by chemical features of the substrate, vegetation composition, and water depth Knowledge on the fauna contributes to the assessment of water quality and development of biodiversity conservation activities Different biotic factors affecting the invertebrate community were evaluated in the Alagados reservoir, the main water source of the city of Ponta Grossa, Paraná. In five different sampling points, 18,473 specimens of aquatic or semiaquatic macroinvertebrates were collected, belonging to 46 taxa of the phylla Annelida (Hirudinea and Oligochaeta), Mollusca (Gastropoda), Platyhelminthes (Turbellaria), Nematoda and Arthropoda (Arachnida, Crustacea and Insecta). This community was composed mainly of predators ( $45.7 \%$ of the taxa sampled), collectors and/or filterers (23.9\%), scrapers (15.2\%), shredders (13.0\%) and detritivores $(2.2 \%)$. Diversity ( $\left.\mathrm{H}^{\prime}\right)$ and evenness $(\mathrm{J})$ indices were significantly low for the sites examined, and $\mathrm{H}^{\prime}$ ranged between 0.3301 and 1.0396 . Regarding tolerance of organisms to organic pollution, more sensitive taxa were very rare (Plecoptera) or unusual (Trichoptera and Ephemeroptera). Among the more resistant groups are Chironomidae and Hirudinea, both fairly common in the samples. This study corroborates the importance of bioindicators as a tool to assess water quality for human consumption and for the conservation of aquatic environments, integrating physical, chemical and biological factors in monitoring programs.
\end{abstract}

KEYWORDS. Bioindicators, environmental quality, biotic indices, eutrophication.

RESUMO. Os macroinvertebrados bentônicos e nectônicos representam elementos importantes na estrutura e funcionamento dos ecossistemas aquáticos e sua distribuição é influenciada pela natureza química do substrato, composição da vegetação e profundidade da lâmina d'água. O conhecimento desta fauna contribui para a avaliação da qualidade da água e a elaboração de ações visando à conservação da biodiversidade. No presente estudo foram avaliadas diferentes medidas bióticas da comunidade de invertebrados da represa de Alagados, importante manancial da cidade de Ponta Grossa, no Paraná. Em cinco diferentes pontos de amostragem, foram coletados 18.473 exemplares de macroinvertebrados aquáticos ou semi-aquáticos, pertencentes a 46 táxons dos filos Annelida (Hirudinea e Oligochaeta), Mollusca (Gastropoda), Platyhelminthes (Turbellaria), Nematoda e Arthropoda (Arachnida, Crustacea e Insecta). Esta comunidade foi constituída predominantemente por organismos predadores (45,7\% dos táxons amostrados), seguidos de coletores e/ou filtradores (23,9\%); raspadores $(15,2 \%)$, fragmentadores (13\%) e detritívoros $(2,2 \%)$. De modo geral, os índices de diversidade (H') e equitabilidade (J) foram significativamente baixos para os cinco locais investigados, com H' variando de 0,3301 a 1,0396. Quanto à tolerância dos organismos à poluição orgânica, alguns táxons mais sensíveis foram muito raros (Plecoptera) ou em baixa frequência (Trichoptera e Ephemeroptera). Entre os grupos mais resistentes a ambientes poluídos estão os Chironomidae e os Hirudinea, ambos bastante comuns nas amostras de Alagados. Este estudo reforça a importância da análise de bioindicadores na avaliação da qualidade de água para consumo humano e também para a conservação de ecossistemas, considerando que um programa de monitoramento ambiental deve integrar medidas físicas, químicas e biológicas.

PALAVRAS-CHAVE. Bioindicadores, qualidade ambiental, índices bióticos, eutrofização.

Os macroinvertebrados aquáticos bentônicos e nectônicos representam elementos importantes na estrutura e funcionamento dos ecossistemas aquáticos e sua distribuição é influenciada por padrões biogeográficos regionais e locais, como a composição da vegetação, profundidade da lâmina d'água, natureza química do substrato, concentração de oxigênio e disponibilidade de alimento, entre outros (QueIroz et al., 2000; Oliveira et al., 2005; HeINo, 2009).

Em um lago ou uma represa, a fauna de invertebrados pode habitar três áreas distintas: a região litorânea, com poucos metros de profundidade e que geralmente está relacionada à presença de macrófitas; a zona profunda, desprovida de vegetação, geralmente conhecida como 
região limnética, e uma terceira, não tão bem delimitada, e que corresponde a uma área de transição entre as duas anteriores, denominada de sublitorânea. A diversidade e a riqueza dos grupos que habitam as regiões mais rasas (litorânea e sublitorânea) são frequentemente elevadas, principalmente devido à influência da mata ripária, os efeitos climáticos e a presença de macrófitas aquáticas nas suas margens que pode determinar a maior abundância de microhábitats (MANDAVILLE, 2002).

Com relação à fauna de invertebrados aquáticos, tem-se observado que a eutrofização pode causar o aumento da biomassa de alguns grupos taxonômicos, sobretudo Oligochaeta (ZinCHENKo, 1992) e Chironomidae (DoRnFeld \& FonseCA-GESSNER, 2005), a diminuição da diversidade (VAlenti \& Froehlich, 1986), a reestruturação das comunidades com o desaparecimento de espécies sensíveis e o surgimento daquelas mais tolerantes às novas condições, entre outros efeitos (PAmplin et al., 2006; PAmplin \& Rocha, 2007). Estudos desta fauna contribuem para uma melhor compreensão dos sistemas aquáticos, no que se refere à avaliação da qualidade da água e a elaboração de ações visando a conservação da biodiversidade local (CALLISTO et al., 2001; GonçALVES \& ARANHA, 2004).

Em Ponta Grossa, toda a água que abastece a cidade provém do rio Pitangui e seus tributários, sendo que cerca de $30 \%$ deste total é originário da represa de Alagados. Apesar da grande importância para a população, a represa tem sofrido inúmeras agressões por meio da contaminação com dejetos humanos e de suínos (decorrentes dos vários criadouros próximos), da lixiviação de fertilizantes e agrotóxicos e do desmatamento de suas margens (Moro et al., 2003; SEPKA et al., 2005; TARDIVO et al., 2007).

O presente estudo visou realizar uma caracterização qualitativa e quantitativa da fauna de invertebrados bentônicos e nectônicos de Alagados, na região de Ponta Grossa, Paraná. Pelas características deste corpo d'água, que recebe considerável aporte de sedimentos e poluentes orgânicos, espera-se encontrar uma comunidade que reflita seu estado de degradação, com dominância de alguns grupos mais resistentes, e que apresente uma menor diversidade biológica quando comparado com ambientes mais íntegros, avaliados em outros estudos. Assim, procurou-se responder as seguintes questões: 1. qual a estrutura e a composição taxonômica dos macroinvertebrados, tendo em vista que essa comunidade ocorre em um ambiente eutrofizado? 2. há uma correlação entre a distribuição de abundância, a caracterização funcional e as condições abióticas da água? 3. a composição e a diversidade faunística são comparáveis com as de outros ambientes lênticos no Brasil?

\section{MATERIAL E MÉTODOS}

O manancial de Alagados é formado pelo represamento do rio Pitangui, afluente do rio Tibagi, e localiza-se entre as coordenadas $24^{\circ} 52^{\prime}$ a $25^{\circ} 05^{\prime} \mathrm{S}$ e $49^{\circ} 46^{\prime}$ a 5006'W nos limites dos municípios de Ponta Grossa, Castro e Carambeí, no estado do Paraná. Apresenta uma extensão aproximada de $15 \mathrm{~km}$, largura média de 500 m e profundidade variando de $15 \mathrm{~m}$, na região próxima à barragem, a 2-5 m, no restante da área. A região apresenta clima tipo $\mathrm{Cfb}$ (mesotérmico úmido com verão temperado), sem estação seca definida, segundo a classificação de Köppen. A precipitação anual média é de 1400 a 1600 mm, sendo que junho a agosto compreende o período mais seco, com total médio de 250 a $350 \mathrm{~mm}$, e dezembro a fevereiro os meses mais chuvosos, com total médio de 500 a $600 \mathrm{~mm}$ (Moro et al., 2005). Os valores de precipitação anual e mensal referentes ao período em que foi realizada a pesquisa estão um pouco abaixo das médias históricas para a região, tendo sido registrados $1380 \mathrm{~mm}$ de precipitação anual, com total de $210 \mathrm{~mm}$ de chuva para os meses de junho a agosto e de $350 \mathrm{~mm}$ para os meses de dezembro a fevereiro (FUnDAÇão ABC, 2008).

A microbacia de Alagados encontra-se em uma área de transição entre o primeiro e o segundo planalto paranaense (Moro et al., 2005). Na bacia do rio Pitangui, a montante do reservatório, as grandes propriedades priorizam a agricultura mecanizada, a pecuária e a monocultura florestal com espécies exóticas, enquanto que nas pequenas propriedades predominam a suinocultura e avicultura de corte. A represa é também utilizada para o lazer, existindo em suas margens casas de veraneio e dois clubes náuticos (Moro et al., 2003).

Mensalmente, de março de 2005 a fevereiro de 2006, foram realizadas coletas em cinco pontos das áreas marginais da represa, onde ocorre maior acúmulo de macrófitas. Estes pontos foram estabelecidos buscando diagnosticar as variações ambientais, bem como os diferentes níveis de impactação deste reservatório: ponto 1 - alto do reservatório, trecho mais lótico, recebe as águas do rio Jutuva, que transporta rejeitos domésticos e agropecuários do município de Carambeí; pontos 2 e 3 trechos lênticos, próximos a habitações e margens com agricultura; ponto 4 - trecho mais lótico do Pitangui, no início do represamento deste rio, margens com mata ripária; ponto 5 - trecho lêntico, próximo à desembocadura de um pequeno tributário, da barragem e do local de captação, margens com reflorestamento de Pinus sp. Nos meses de setembro e outubro de 2005, em que o nível da água estava muito baixo, por conta de manutenção da represa pela companhia de saneamento, não foi possível realizar as coletas.

No levantamento dos macroinvertebrados foram utilizados "puçás aquáticos" de $0,15 \mathrm{~m}^{2}$ de área e malha de $300 \mu \mathrm{m}$, para coleta de duas amostras de sedimento em cada ponto. Foram também recolhidos os organismos nectônicos observados no momento da coleta, além de amostras de raízes e outras partes submersas das plantas aquáticas. Todo o material recolhido, juntamente com um pouco de água, foi acondicionado, em vasilhames plásticos com capacidade de três litros. Para cada local correspondia um vasilhame. As amostras foram identificadas segundo o ponto e a data de coleta. No dia seguinte à coleta, no laboratório, o conteúdo de cada vasilhame foi lavado em peneira também de malha $300 \mu \mathrm{m}$, para, em seguida, os organismos serem triados em bandejas transiluminadas e fixados em álcool $80 \%$. Posteriormente, os exemplares foram observados em estereomicroscópio e identificados com auxílio de bibliografia especializada. 
Os insetos capturados foram identificados no nível taxonômico de família e os demais grupos no nível de subordem (Prostigmata), classe (Hirudinea, Oligochaeta, Tricladida, Gastropoda, Amphipoda, Cladocera, Copepoda e Ostracoda) e filo (Nematoda). A identificação dos exemplares foi realizada baseando-se em MCCAFFERTY (1981), MerritT \& Cummins (1996), Trivinho-Strixino \& Strixino (1995), Nieser \& Melo (1997), Carvalho \& Calil (2000), Fernández \& Domínguez (2001). O materialtestemunho está depositado na Coleção Entomológica dos Campos Gerais do Paraná, Departamento de Biologia Geral, Universidade Estadual de Ponta Grossa.

Alguns parâmetros físicos e químicos, como temperatura, $\mathrm{pH}$, alcalinidade total, turbidez e oxigênio dissolvido são apresentados com o intuito de conhecer os efeitos da ocupação da bacia sobre a qualidade das águas do reservatório e também para subsidiar o estudo da macrofauna de invertebrados. Tais dados, fornecidos pela Companhia de Água e Saneamento do Paraná (SANEPAR), são oriundos de amostras coletadas diariamente em um único local da represa (situado próximo à barragem), o que impossibilita uma análise mais detalhada das respostas da fauna de macroinvertebrados nos diferentes pontos de amostragem, frente a estes dados ambientais. Os testes ANOVA de uma via e T (uma amostra) foram usados para determinar diferenças entre as amostras mensais para as variáveis abióticas e os macroinvertebrados. Para identificar quais destas variáveis influenciariam na ocorrência dos macroinvertebrados, foi realizada uma análise de regressão múltipla. Para estes testes foi utilizado o software livre BioEstat versão 5.0.

A abundância relativa e a frequência de ocorrência (constância) dos táxons foram calculadas e categorizadas segundo JablonsKa \& Patures (1999) e PAMplin et al. (2005).

Os dados da comunidade de invertebrados foram analisados por meio do número total de indivíduos e de famílias, além do cálculo de algumas medidas bioindicadoras como \% de Chironomidae, \% de EPT (Ephemeroptera, Plecoptera e Trichoptera), \% de fragmentadores, índice de riqueza de Margalef $(D \alpha)$, índice de diversidade de ShannonWiener $\left(\mathrm{H}^{\prime}\right)$, de uniformidade ou equitabilidade de Pielou (J), índice biótico BMWP - Biological Monitoring Work Party - (JunqueIRA \& CAMPos, 1998, JunqueIRA et al., 2000) e índice ASPT - Average Score Per Taxon - (Mandaville, 2002; BAPTISTA, 2008).
As categorias tróficas funcionais foram estabelecidas de acordo com a classificação de Cummins \& Klug (1979) e MerRITT \& Cummins (1996), sendo a fauna subdividida nas categorias: fragmentadores, incluindo comedores de material vegetal alóctone e autóctone; coletores, incluindo filtradores; raspadores de perifíton (diatomáceas, outras algas e fungos), detritívoros e predadores.

\section{RESULTADOS}

Variáveis físicas e químicas. As temperaturas médias do ar variaram significativamente ao longo do ano $(F=15,72 ; p<0,001 ; G . L$.tratamento $=9 ; G . L$.resíduo $=272)$, 0 mesmo tendo sido verificado em relação às temperaturas da água $(\mathrm{F}=135,2 ; \mathrm{p}<0,001 ;$ G.L.tratamento $=$ 9; G.L.resíduo $=272$ ). As temperaturas máximas e mínimas da água foram registradas em janeiro e agosto, respectivamente, enquanto que as máximas e mínimas do ar observadas em março e agosto, indicando uma relação entre o regime térmico e a sazonalidade local. Em janeiro foi observada a maior precipitação entre os meses em que foram realizadas coletas (Tab. I).

As concentrações de O.D. mostraram variações ao longo do ano $(t=8,508 ; \mathrm{p}<0,001)$, sendo os valores máximo e mínimo registrados nos meses de junho e dezembro, respectivamente (Tab. I).

As águas de Alagados apresentaram características alcalinas na maior parte do ano, com valores médios das máximas e mínimas de $\mathrm{pH}$ na faixa entre 6,9 a 7,5 e ligeira diferença entre os períodos de chuva e de estiagem $(\mathrm{F}=$ 9,741; p < 0,001; G.L.tratamento $=9$; G.L.resíduo $=552)($ Tab. I) .

Com relação à alcalinidade total, as médias das máximas e mínimas diferiram ao longo do período $(\mathrm{F}=$ 40,22; p < 0,001; G.L.tratamento $=9 ;$ G.L. resíduo $=482$ ), sendo que os valores mais elevados foram obtidos nos meses de janeiro, fevereiro e agosto (Tab. I).

A turbidez, que também variou significativamente no período $(F=192,4 ; p<0,001 ; G . L$.tratamento $=9 ; G . L$.resíduo =276), atingiu valores máximos nos meses de novembro e agosto (Tab. I), coincidindo com a época de menor precipitação e com a diminuição da profundidade da coluna de água da represa, por conta de manutenção no sistema, pela SANEPAR.

Macroinvertebrados. Foram coletados 18.473 macroinvertebrados aquáticos ou semi-aquáticos,

Tabela I. Dados de precipitação, alguns parâmetros físicos e químicos das águas, abundância (N) e riqueza de macroinvertebrados (S) de Alagados, Paraná, no período de março de 2005 a fevereiro de 2006 (precipitação acumulada (mm de chuva); valores das médias mensais e desvio padrão da temperatura do ar e da água $\left({ }^{\circ} \mathrm{C}\right) ; \mathrm{pH}$; turbidez $\left(\mathrm{mg} / 1 \mathrm{SiO}_{2}\right)$; alcalinidade total $\left(\mathrm{mgCaCO}_{3} / \mathrm{l}\right)$; O.D., oxigênio dissolvido (mg/l): valor de uma amostra).

\begin{tabular}{lcccccccccc}
\hline & Mar & Abr & Mai & Jun & Jul & Ago & Nov & Dez & Jan & Fev \\
\hline Precipitação & 70,0 & 130,0 & 110,0 & 90,0 & 70,0 & 50,0 & 70,0 & 70,0 & 170,0 & 110,0 \\
$\mathrm{~T}{ }^{\circ} \mathrm{C}$ do ar & $20,2 \pm 2,6$ & $18,1 \pm 3,6$ & $15,1 \pm 2,7$ & $14,9 \pm 2,2$ & $14,8 \pm 2,4$ & $14,5 \pm 3,5$ & $16,9 \pm 3,4$ & $17,8 \pm 2,9$ & $20,0 \pm 3,1$ & $17,5 \pm 2,3$ \\
$\mathrm{~T}{ }^{\circ} \mathrm{C}$ da água & $22,2 \pm 0,6$ & $21,1 \pm 1,1$ & $18,2 \pm 0,8$ & $17,0 \pm 0,7$ & $17,0 \pm 0,7$ & $15,2 \pm 0,9$ & $19,3 \pm 0,9$ & $20,3 \pm 0,9$ & $23,8 \pm 3,1$ & $22,9 \pm 0,9$ \\
$\mathrm{O} . \mathrm{D}$. & 6,4 & 4,7 & 6,6 & 11,6 & 4,8 & 9,0 & 5,5 & 4,2 & 4,6 & 5,4 \\
$\mathrm{pH}$ med. & $7,3 \pm 0,1$ & $7,4 \pm 0,1$ & $7,2 \pm 0,1$ & $7,2 \pm 0,1$ & $7,2 \pm 0,1$ & $7,5 \pm 0,2$ & $6,9 \pm 1,1$ & $7,3 \pm 0,2$ & $7,4 \pm 0,2$ & $7,5 \pm 0,3$ \\
Turbidez & $3,3 \pm 0,3$ & $3,5 \pm 0,4$ & $3,9 \pm 0,3$ & $4,1 \pm 0,3$ & $4,1 \pm 0,4$ & $11,6 \pm 3,6$ & $15,1 \pm 1,9$ & $7,6 \pm 2,2$ & $3,8 \pm 0,6$ & $3,9 \pm 0,5$ \\
Alcalinidade total & $15,9 \pm 1,3$ & $16,3 \pm 3,7$ & $17,5 \pm 2,2$ & $18,0 \pm 2,9$ & $18,1 \pm 3,0$ & $19,6 \pm 1,5$ & $16,5 \pm 2,1$ & $18,8 \pm 1,7$ & $22,7 \pm 3,9$ & $23,0 \pm 2,9$ \\
$\mathrm{~N}^{\circ}$ indiv. (N) & 542 & 1826 & 1126 & 478 & 864 & 262 & 1338 & 1486 & 6407 & 4144 \\
$\mathrm{~N}^{\circ}$ táxons (S) & 28 & 21 & 26 & 27 & 31 & 23 & 29 & 28 & 36 & 33 \\
\hline
\end{tabular}


pertencentes a 46 táxons dos filos Annelida (Hirudinea e Oligochaeta), Mollusca (Gastropoda), Platyhelminthes (Turbellaria), Nematoda e Arthropoda (Arachnida, Crustacea e Insecta) (Tab. II). As ordens com maior número de famílias foram: Coleoptera (10), Hemiptera (7) e Diptera (6).

Comparando os trechos amostrados, a maior riqueza taxonômica, em números absolutos, foi observada no ponto 2, totalizando 38 táxons, sendo janeiro e fevereiro os meses com maior número de táxons neste local (26 e 22, respectivamente) (Fig. 1). A menor riqueza absoluta foi verificada no ponto 5 , com 31 táxons, sendo registrados somente cinco grupos no mês de maio (Fig. 1). Quanto ao índice de riqueza de Margalef ( $\mathrm{D} \alpha)$, este foi mais elevado no ponto 4 e mais baixo no ponto 3 (Tab. III).

Ao todo, 23 grupos foram comuns aos cinco locais de coleta, dos quais Chironomidae e Hirudinea foram os mais abundantes, enquanto que seis táxons foram exclusivos a um determinado ponto, além de aparecerem em baixa frequência, como Gripopterygidae (Plecoptera), Gerridae, Hydrometridae e Nepidae (Hemiptera) e Chrysomelidae (Coleoptera).

A avaliação de dominância (Tab. II) revelou a presença de um táxon dominante, Chironomidae, que constituiu $68 \%$ da comunidade de macroinvertebrados amostrada; um táxon comum, Hirudinea e quatro ocasionais, Hydrophilidae, Dytiscidae, Coenagrionidae e Amphipoda; os demais grupos (40) aparecem como raros, com menos de $1 \%$ do total de exemplares capturados.

Com relação à constância dos grupos nas amostras (Tab. II) observou-se a presença de cinco táxons euconstantes, sendo eles: Chironomidae (presente em 90\% das amostras), Hirudinea morfotipo 1 (86\%), Coenagrionidae (70\%), Dytiscidae e Hydrophilidae (ambos em $66 \%$ das amostras). Do restante dos táxons, sete foram considerados constantes, com destaque para Stratiomyidae (60\%), Amphipoda (56\%), Hirudinea morfotipo 2 e Scirtidae (ambos em 48\%); 15 acessórios, dentre eles Libellulidae, Caenidae e Tabanidae (cada uma em $40 \%$ das coletas), Baetidae (38\%); Oligochaeta (36\%) e Culicidae (36\%) e 19 grupos acidentais, entre os quais os Gastropoda morfotipo 1 e morfotipo 2 (ambos em 18\%) e boa parte dos insetos, tais como as famílias Hydroptilidae (6\%), Notonectidae (6\%), Elmidae e Hydraenidae (ambas em 4\%).

Quanto à sazonalidade dos macroinvertebrados de Alagados, verificou-se atividade durante todo o período de amostragem, porém com maior frequência de ocorrência tanto de táxons $(\mathrm{t}=20,07$ e $\mathrm{p}<0,001)$ quanto de indivíduos $(\mathrm{t}=3,004$ e $\mathrm{p}=0,0148)$ nos meses de janeiro e fevereiro. Porém, para vários grupos, pelo número reduzido de exemplares capturados, não foi possível fazer inferências sobre sua atividade sazonal. Chironomidae e Hirudinea foram os táxons mais frequentes, presentes em quase todas as amostras.

Resultados de regressão múltipla indicam que a riqueza dos macroinvertebrados (número de táxons) em Alagados foi influenciada mais fortemente pela alcalinidade total $(b=2,435 ; p=0,023), p H(b=-25,203 ; p$ $=0,031)$ e turbidez $(\mathrm{b}=-0,612 ; \mathrm{p}=0,086)$, e fracamente influenciada pela temperatura do $\operatorname{ar}(b=1,939 ; p=0,160)$, precipitação $(b=-0,037 ; p=0,238)$, temperatura da água $(b=-1,051 ; p=0,321)$ e teor de oxigênio $(b=-0,282 ; p=$ $0,444)$, sendo o modelo de regressão significativo ( $\mathrm{R}^{2}$ ajustado $=0,871 ; p=0,055)$. Para a abundância de espécimes, o modelo de regressão $\left(R^{2}\right.$ ajustado $=0,980 ; p$ $=0,007)$ foi definido por alcalinidade total $(\mathrm{b}=492,32 ; \mathrm{p}=$ $0,016)$ e precipitação $(b=26,01 ; p=0,020)$ e mais fracamente por $\mathrm{pH}(\mathrm{b}=-1712,03 ; \mathrm{p}=0,160)$, oxigênio $(\mathrm{b}=-102,31 ; \mathrm{p}=$ $0,181)$, temperatura do ar $(b=252,48 ; p=0,236)$ e turbidez $(b=39,41 ; p=0,353)$.

Quanto à contribuição de cada grupo trófico para a composição da comunidade de Alagados, 21 táxons foram classificados como predominantemente predadores $(45,6 \%), 11$ como coletores e/ou filtradores $(23,9 \%)$, sete como raspadores $(15,2 \%)$, seis como fragmentadores $(13,0 \%)$ e um como detritívoro (2,2\%) (Tab. II). Vários destes, no entanto, são generalistas oportunistas quanto ao hábito alimentar, ou seja, participam de mais de uma categoria trófica, conforme a disponibilidade de alimento.

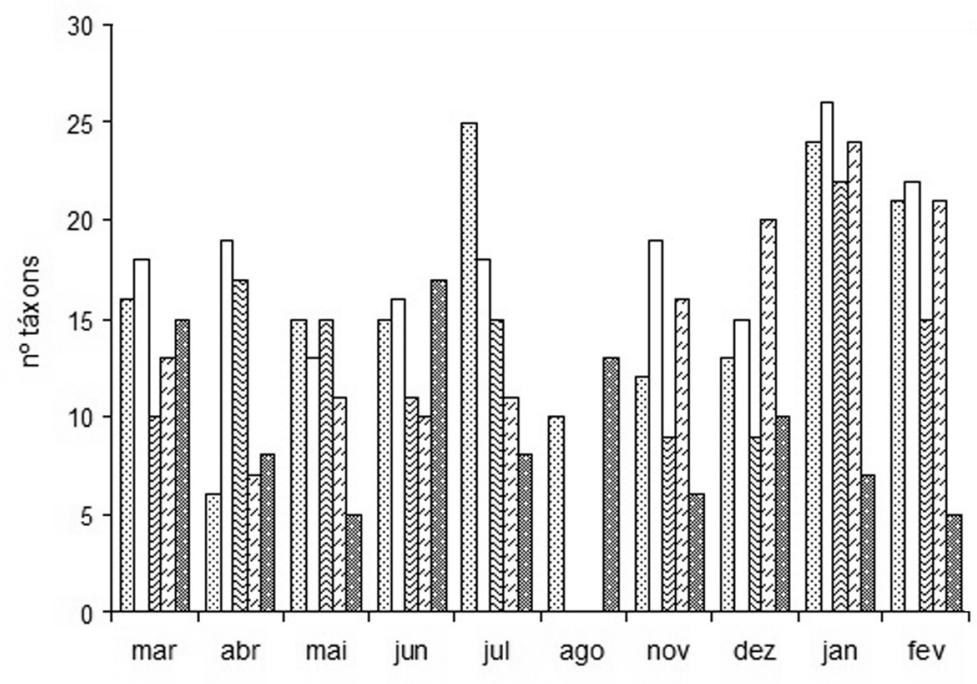

图 ponto $1 \square$ ponto 2 圈ponto 3 ponto 4 ponto 5

Figura 1. Riqueza de táxons de macroinvertebrados registrada para a represa de Alagados, na região de Ponta Grossa, Paraná, no período de março de 2005 a fevereiro de 2006. 
Tabela II. Macroinvertebrados aquáticos coletados em cinco pontos de amostragem na represa de Alagados, Paraná, no período de março de 2005 a fevereiro de 2006 (dominante (Dom): > 50\%; comum (Com): 10-29,9\%; ocasional (Oca): 1-9,9\%; raro (Rar): < 1\%; euconstante $(\mathrm{Eu}):>61 \%$; constante $(\mathrm{Ct})$ : 41-61\%; acessório (As): 21-40\%; acidental (Ac): < 20\%. Tolerância do táxon à poluição orgânica (score): de 1, muito tolerante a 10, intolerante. Scores* baseados em JunQuEIRA \& CAMPOS (1998) e JunQuEIRA et al. (2000).

\begin{tabular}{|c|c|c|c|c|c|c|c|c|c|c|c|}
\hline Táxon & $\begin{array}{l}\text { Categorias } \\
\text { tróficas } \\
\text { funcionais }\end{array}$ & Ponto 1 & Ponto 2 & Ponto 3 & Ponto 4 & Ponto 5 & 5 Total & $\begin{array}{c}\text { Abund. I } \\
\text { Relat. }(\%)\end{array}$ & Dominância & Constância & Score* \\
\hline $\begin{array}{l}\text { Platyhelminthes } \\
\text { Turbellaria } \\
\text { Tricladida sp. } 1\end{array}$ & Predador & 52 & 19 & 43 & 5 & & 119 & 0,64 & Rar & Ac & 7 \\
\hline $\begin{array}{c}\text { Nematoda sp.1 } \\
\text { Mollusca }\end{array}$ & Predador & 5 & 8 & 9 & & 8 & 30 & 0,16 & Rar & Ac & Indet. \\
\hline Gastropoda morfotipo & Raspador & 12 & 3 & & 20 & & 35 & 0,19 & Rar & $\mathrm{Ac}$ & 4 \\
\hline Gastropoda morfotipo & Raspador & 5 & 3 & 3 & 45 & & 56 & 0,30 & Rar & Ac & 4 \\
\hline $\begin{array}{l}\text { Gastropoda morfotipo } \\
\text { Annelida }\end{array}$ & Raspador & & 19 & 6 & & & 25 & 0,13 & Rar & Ac & 4 \\
\hline Hirudinea morfotipo 1 & Predador & 1224 & 760 & 249 & 88 & 76 & 2397 & 12,98 & Com & $\mathrm{Eu}$ & 1 \\
\hline Hirudinea morfotipo 2 & Predador & 27 & 17 & 12 & 8 & 2 & 66 & 0,36 & Rar & $\mathrm{Ct}$ & 1 \\
\hline $\begin{array}{l}\text { Oligochaeta } \\
\text { Arthropoda } \\
\text { Crustacea }\end{array}$ & Detritívoro & 56 & 25 & 5 & 5 & 20 & 111 & 0,60 & Rar & As & 1 \\
\hline Amphipoda & Raspador & 117 & 39 & 16 & 8 & 7 & 187 & 1,01 & Oca & $\mathrm{Ct}$ & 4 \\
\hline Cladocera & Raspador & 58 & 74 & 1 & 8 & & 141 & 0,76 & Rar & Ac & 4 \\
\hline Copepoda & Coletor/Filtrador & 4 & 4 & & & & 8 & 0,04 & Rar & Ac & 4 \\
\hline $\begin{array}{l}\text { Ostracoda } \\
\text { Collembola }\end{array}$ & Coletor & 42 & 24 & 2 & 10 & & 78 & 0,42 & Rar & As & 4 \\
\hline $\begin{array}{l}\text { Poduridae } \\
\text { Insecta } \\
\text { Odonata }\end{array}$ & Fragmentador & 26 & & & 1 & & 27 & 0,15 & Rar & Ac & 7 \\
\hline Coenagrionidae & Predador & 108 & 132 & 66 & 31 & 64 & 401 & 2,17 & Oca & $\mathrm{Eu}$ & 6 \\
\hline $\begin{array}{l}\text { Libellulidae } \\
\qquad \text { Ephemeroptera }\end{array}$ & Predador & 11 & 10 & & 17 & 12 & 50 & 0,27 & Rar & As & 8 \\
\hline Baetidae & Coletor & 42 & 80 & 16 & 6 & 11 & 155 & 0,84 & Rar & As & 5 \\
\hline $\begin{array}{l}\text { Caenidae } \\
\text { Plecoptera }\end{array}$ & Coletor & 27 & 12 & 6 & 3 & 83 & 131 & 0,71 & Rar & As & 7 \\
\hline $\begin{array}{l}\text { Gripopterygidae } \\
\text { Trichoptera }\end{array}$ & Coletor & 1 & & & & & 1 & 0,005 & Rar & Ac & 10 \\
\hline Hydroptilidae & Raspador & 1 & 1 & & 2 & & 4 & 0,02 & Rar & Ac & 6 \\
\hline $\begin{array}{c}\text { Polycentropodidae } \\
\text { Hemiptera }\end{array}$ & Coletor/Predador & 4 & 12 & 5 & 29 & 16 & 66 & 0,36 & Rar & As & 7 \\
\hline Belostomatidae & Predador & 4 & 7 & 4 & 12 & 4 & 31 & 0,17 & Rar & As & 5 \\
\hline Corixidae & Predador & 42 & 2 & 77 & 52 & 2 & 175 & 0,95 & Rar & As & 5 \\
\hline Gerridae & Predador & & & & & 1 & 1 & 0,005 & Rar & Ac & 5 \\
\hline Hydrometridae & Predador & & & & & 2 & 2 & 0,01 & Rar & Ac & 3 \\
\hline Nepidae & Predador & & & & & 4 & 4 & 0,02 & Rar & Ac & 6 \\
\hline Notonectidae & Predador & 4 & & & 7 & 1 & 12 & 0,06 & Rar & $\mathrm{Ac}$ & 7 \\
\hline $\begin{array}{l}\text { Vellidae } \\
\text { Lepidoptera }\end{array}$ & Predador & 4 & 2 & 3 & 1 & 4 & 14 & 0,08 & Rar & As & 7 \\
\hline Noctuidae & Fragmentador & 4 & 6 & 6 & 7 & 3 & 26 & 0,14 & Rar & As & 8 \\
\hline $\begin{array}{l}\text { Pyralidae } \\
\text { Coleoptera }\end{array}$ & Fragmentador & 2 & 3 & 6 & 10 & 7 & 28 & 0,15 & Rar & As & 8 \\
\hline Chrysomelidae & Fragmentador & & & & & 1 & 1 & 0,005 & Rar & $\mathrm{Ac}$ & 4 \\
\hline Curculionidae & Fragmentador & & 1 & & 5 & & 6 & 0,03 & Rar & Ac & 5 \\
\hline Dytiscidae & Predador & 43 & 67 & 116 & 40 & 14 & 280 & 1,52 & Oca & $\mathrm{Eu}$ & 4 \\
\hline Elmidae & Coletor & & & 1 & & 1 & 2 & 0,01 & Rar & Ac & 5 \\
\hline Hydraenidae & Raspador/Predador & & 1 & & 8 & & 9 & 0,05 & Rar & $\mathrm{Ac}$ & 5 \\
\hline Hydrophilidae & Fragmentador/Coletor & or 68 & 88 & 38 & 222 & 9 & 425 & 2,30 & Oca & $\mathrm{Eu}$ & 5 \\
\hline Lampyridae & Predador & 5 & 18 & 16 & 32 & & 71 & 0,38 & Rar & $\mathrm{Ct}$ & Indet. \\
\hline Noteridae & Predador & & 3 & & & & 3 & 0,02 & Rar & Ac & 4 \\
\hline Scirtidae & Coletor/Filtrador & 9 & 40 & 22 & 24 & 21 & 116 & 0,63 & Rar & $\mathrm{Ct}$ & 7 \\
\hline $\begin{array}{r}\text { Staphylinidae } \\
\text { Diptera }\end{array}$ & Predador & 2 & 20 & 10 & 4 & 1 & 37 & 0,20 & Rar & As & 7 \\
\hline Ceratopogonidae & Predador & 24 & 68 & 18 & 8 & 17 & 135 & 0,73 & Rar & $\mathrm{Ct}$ & 4 \\
\hline Chaoboridae & Predador & 24 & 16 & 9 & 12 & & 61 & 0,33 & Rar & As & 8 \\
\hline Chironomidae & Coletor/Predador & 356 & 5797 & 5114 & 531 & 773 & 12571 & 68,05 & Dom & $\mathrm{Eu}$ & 2 \\
\hline Culicidae & Coletor & 26 & 19 & 9 & 12 & 5 & 71 & 0,38 & Rar & As & 2 \\
\hline Stratiomyidae & Coletor & 14 & 49 & 34 & 25 & 10 & 132 & 0,71 & Rar & $\mathrm{Ct}$ & 2 \\
\hline $\begin{array}{l}\text { Tabanidae } \\
\text { Arachnida }\end{array}$ & Predador & 18 & 21 & 10 & 9 & 2 & 60 & 0,32 & Rar & As & 3 \\
\hline Acarina Prostigmata & Predador & 6 & 27 & 9 & 46 & 24 & 112 & 0,61 & Rar & $\mathrm{Ct}$ & 3 \\
\hline
\end{tabular}


Tabela III. Medidas bioindicadoras da represa de Alagados, Paraná ( $\mathrm{N}, \mathrm{n}^{\circ}$ total de indivíduos; $\mathrm{S}, \mathrm{n}^{\circ}$ total de táxons; D $\alpha$, índice de riqueza de Margalef; H', índice de diversidade de Shannon-Wiener; J, índice de equitabilidade de Pielou; *, \% de Ephemeroptera + Plecoptera + Trichoptera; **, índice BMWP (Biological Monitoring Work Party) calculado para o presente estudo, segundo JunQuEIRA \& CAMPOS (1998) e JunQueIra et al. (2000); ***, índice ASTP (Average Score Per Taxon), segundo Mandaville (2002) e BaPTisTa (2008), BMWP/nº táxons).

\begin{tabular}{|c|c|c|c|c|c|}
\hline & Ponto 1 & Ponto 2 & Ponto 3 & Ponto 4 & Ponto 5 \\
\hline $\mathrm{N}$ & 2477,000 & 7497,000 & 5941,000 & 1353,000 & 1205,000 \\
\hline $\mathrm{S}$ & 37,000 & 38,000 & 32,000 & 36,000 & 31,000 \\
\hline $\mathrm{D} \alpha$ & 10,607 & 9,549 & 8,214 & 11,177 & 9,737 \\
\hline $\mathrm{H}^{\prime}$ & 0,908 & 0,465 & 0,330 & 1,039 & 0,699 \\
\hline $\mathrm{J}$ & 0,579 & 0,295 & 0,219 & 0,668 & 0,469 \\
\hline$\%$ Chironomidae & 14,400 & 77,300 & 86,100 & 39,200 & 64,100 \\
\hline$\% \mathrm{EPT}^{*}$ & 3,000 & 1,400 & 0,500 & 3,000 & 9,100 \\
\hline$\%$ fragmentadores & 4,000 & 1,000 & 0,800 & 18,100 & 2,000 \\
\hline Índice biótico BMWP** & 177,000 & 171,000 & 145,000 & 177,000 & 143,000 \\
\hline Índice ASPT**** & 4,800 & 4,500 & 4,500 & 4,900 & 4,600 \\
\hline
\end{tabular}

Sobre a ocorrência dos grupos tróficos ao longo do trecho amostrado, verificou-se que os coletores e/ou filtradores foram os mais frequentes nas amostras dos pontos 2, 3, 4 e 5 (representando 80,5\%; 87,7\%; 47,3\% e $76,3 \%$ respectivamente) seguido dos predadores $(16,0 \%$; $11,0 \% ; 27,5 \%$ e $19,7 \%$ ); enquanto que no ponto 1 , estas proporções se invertem $(64,7 \%$ para os predadores e $21,2 \%$ para os coletores e/ou filtradores). Os fragmentadores foram mais abundantes nos pontos 4 e 1 ( $18,1 \%$ e $4,0 \%$, respectivamente); os raspadores nos pontos $1(7,8 \%)$ e $4(6,7 \%)$ e os detritívoros nos pontos $1(2,3 \%)$ e $5(1,7 \%)$.

As porcentagens de EPT foram baixas em todos os pontos avaliados (Tabs II, III), sendo a maior taxa observada no ponto 5 , apesar do local apresentar algum grau de eutrofização, devido ao aporte de material alóctone (sedimento e matéria orgânica) oriundo de um tributário que deságua próximo e de suas áreas marginais, onde há um reflorestamento de Pinus sp. Os táxons que elevaram este valor foram os efemerópteros das famílias Baetidae e Caenidae, ambos considerados moderadamente tolerantes à poluição (apresentam score 5). As famílias Polycentropodidae e Hydroptilidae representaram a ordem Trichoptera, sendo que a primeira esteve presente em todos os pontos de coleta e em maior frequência. É importante notar que apenas um indivíduo da ordem Plecoptera (em sua maioria, sensíveis a perturbações) foi coletado durante todo o período de estudo (no ponto 1).

Os índices de riqueza $(\mathrm{D} \alpha)$, diversidade $\left(\mathrm{H}^{\prime}\right)$ e equitabilidade (J) foram significativamente baixos para os cinco pontos investigados, aumentando no sentido: pto 3 < pto 2 < pto 5 < pto 1 < pto 4 , enquanto que a porcentagem de Chironomidae decresceu no sentido: pto $3>$ pto $2>$ pto $5>$ pto $4>$ pto 1 (Tab. III).

No que se refere ao índice BMWP, os valores obtidos para os pontos 1, 2 e 4 permitem classificá-los como não poluídos - classe I, enquanto que os trechos 3 e 5 apresentam valores que os classificam como levemente poluídos - classe II (Tab. III). Os valores de ASPT para os cinco pontos de coleta variaram pouco, entre 4,5 e 4,9 (Tab. III), sendo que os trechos 1 e 4 apresentaram os maiores índices, enquanto que os pontos 2 e 3 apresentaram os menores valores.

\section{DISCUSSÃO}

Algumas das variáveis aqui avaliadas estão intrinsecamente relacionadas às flutuações sazonais da região, como variações de temperatura e frequência de chuvas que interferem indiretamente em outros parâmetros ambientais, como teores de oxigênio dissolvido e pH (SCHEFFER \& BUSCH, 2010).

De maneira geral, a qualidade das águas de Alagados atende à legislação - Resolução CONAMA 357 (MMA, 2005), que estabelece os padrões de qualidade da água para consumo humano. Por outro lado, concentrações de oxigênio próximas a $5 \mathrm{mg} / \mathrm{l}^{-1}$, valor mínimo estabelecido pela legislação, foram sistematicamente encontrados nos meses com temperaturas mais elevadas. Esses baixos valores de oxigênio dissolvido, que podem também estar relacionados ao aporte de matéria orgânica no sistema, têm diversas implicações do ponto de vista ambiental como, por exemplo, a dificuldade de manutenção de vida dos organismos aeróbios no ambiente aquático.

Vários fatores ambientais, como profundidade, disponibilidade de alimento, concentração de oxigênio e tipo de substrato têm sido apontados como reguladores da estrutura da comunidade de invertebrados em reservatórios (QueIroz et al., 2000; Oliveira et al., 2005; PAMPLim \& Rocha, 2007; Heino, 2009). Em Alagados, os resultados obtidos da análise de regressão múltipla indicam que alcalinidade e precipitação foram positivamente relacionadas com a abundância dos macroinvertebrados, enquanto que $\mathrm{pH}$ e oxigênio foram negativamente relacionados. Isso pode ser explicado pela habilidade de alguns dos grupos mais abundantes (como Chironomidae e Hirudinea) sobreviverem em ambientes com baixo teor de oxigênio. Outro importante fator que afeta a macrofauna de invertebrados da zona litorânea de reservatórios é a variação da profundidade de água, geralmente determinado pelo padrão anual de chuvas, sendo que o aumento da abundância da comunidade bentônica estaria relacionado à queda no nível da água (PAmplim \& Rocha, 2007). Nesta pesquisa, a maior abundância de macroinvertebrados registrada durante o período chuvoso pode não estar relacionada ao regime sazonal de chuva e sim ao fato das amostras serem oriundas das zonas litorâneas e sublitorâneas, com maior 
acúmulo de matéria orgânica, propícias à ocorrência de uma fauna mais abundante e diversa.

As análises faunísticas indicam uma taxocenose diversificada, constituída de 46 táxons pertencentes aos filos Nematoda, Mollusca, Platyhelminthes, Annelida e Arthropoda e com dominância de alguns grupos, o que se reflete nos índices de diversidade, equitabilidade e dominância. Riqueza semelhante foi verificada por Queiroz et al. (2008) em duas microbacias do rio MogiGuaçu, São Paulo, que amostraram 45 famílias de macroinvertebrados, enquanto que PAmplin \& RochA (2007) observaram valores ligeiramente menores em duas represas no rio Tietê, tendo capturado 36 e 39 táxons. É possível que além do estado de conservação dos ambientes, os diversos tipos de amostragem adotados tenham influenciado nos resultados.

Segundo Mandaville (2002) e Pamplin et al. (2006), frequentemente as regiões mais rasas abrigam comunidades mais diversificadas de invertebrados que as regiões mais profundas. STENERT et al. (2004) citam que diferentes grupos de macroinvertebrados estão associados às comunidades de plantas aquáticas. A preferência de alguns táxons, sobretudo insetos, por áreas com vegetação emergente, submersa ou flutuante, tem sido demonstrada em vários estudos (STENERT et al., 2004; Dornfeld \& FonseCa-Gessner, 2005; Pamplin et al., 2006; PeIró \& Alves, 2006). Esta preferência ocorre, segundo os autores, em função da diversidade de oferta de itens alimentares, com material alóctone (vegetais caídos) e autóctone (perifiton, aderido sobre substratos; macrófitas), além do uso do próprio substrato como local de abrigo, fixação, transporte e reprodução.

Vários dos táxons observados neste estudo, também compõem a fauna bentônica de ambientes similares, e.g. lagoas do médio rio Doce, em Minas Gerais (MARQues et al., 1999), lagoas intermitentes na caatinga paraibana (Souza \& ABílio, 2006) e uma barragem em Pelotas, no Rio Grande do Sul (Piedras et al., 2006). Portanto, Alagados apresenta fauna correspondente ao esperado para locais com águas rasas e de pouca correnteza.

Chironomidae e Hirudinea foram os mais abundantes, predominando em todos os pontos de amostragem. Chironomidae é um dos grupos mais populosos, sendo predominante em vários sistemas estudados (MARQues et al., 1999; PAmplin et al., 2006; Piedras et al., 2006; SouZa \& ABílio, 2006; Pamplin \& Rocha, 2007, entre outros). Segundo Di GiovanNi et al. (1996), este táxon, muitas vezes apresenta-se como dominante, tanto em ambientes lênticos, como lóticos, fato que se deve à tolerância de certas espécies a situações extremas como hipóxia. Para Bubinas \& JAGMiniené (2001), determinados gêneros de Chironomidae, como Chironomus Meigen, 1803, Cryptochironomus Kieffer, 1918, Prodiamesa Kieffer, 1906 e Pelopia Meigen, 1800 podem ser classificados como organismos resistentes à poluição, pois são capazes de colonizar ambientes com baixa concentração de oxigênio. Sendo assim, altas densidades destes gêneros podem evidenciar elevado teor de matéria orgânica no ambiente (MARQUES et al., 1999), o que os tornam eficientes indicadores de degradação ambiental.
Os resultados referentes às porcentagens de EPT nas amostras são bem inferiores aos obtidos por QUEIROZ et al. (2008) em duas microbacias do rio Mogi-Guaçu, porém estão próximos ao esperado, uma vez que as ordens EPT, em geral, são mais comuns em águas lóticas e ricas em oxigênio. Como Alagados constitui um típico ambiente lêntico, por si só, este fato já interfere na presença destes grupos, e assim, os baixos valores de EPT, como resultado isolado, não indica má qualidade da água.

Os índices de diversidade, que variaram de $\mathrm{H}^{\prime}=$ 0,3301 a 1,0396, são significativamente menores que os registrados por PIEDRAS et al. (2006) para a barragem Santa Bárbara, em Pelotas, Rio Grande do Sul, que encontraram valores de H' entre 1,44 e 1,75. Nesta mesma barragem, estudos realizados na década de 1980 registraram índices de diversidade superiores a 2,0 em vários trechos ao longo de sua extensão (PIEDRAs et al., 2006). De acordo com WilHm \& Dorris (1968) e Piedras et al. (2006), índices de diversidade $\mathrm{H}^{\prime}$ menores que 1,0 indicam ambiente fortemente poluído; H' entre 1,0 e 3,0 indica poluição moderada e H' superior a 3,0 indica água não poluída. Segundo estes autores, a redução da diversidade pode ser atribuída à deterioração da qualidade da água, que impossibilita o desenvolvimento de determinados grupos de macroinvertebrados. Margalef (1983), Pinto-Coelho et al. (1999) e Melo \& HePP (2008) também consideram que as medidas de riqueza, diversidade e equitabilidade são parâmetros capazes de fornecer informações relevantes sobre a conservação de um corpo d'água e que ambientes impactados tendem a apresentar pequena diversidade biológica, com dominância de poucas espécies.

As menores porcentagens de EPT somadas às elevadas proporções de Chironomidae e os baixos índices de riqueza (D $\alpha$ ), diversidade (H') e equitabilidade (J) nos pontos 2 e 3 evidenciam eutrofização de origem antrópica e consequente poluição da água nestes trechos do reservatório. A baixa correnteza nestes locais determina maior retenção de materiais e poluentes, comprometendo ainda mais a integridade do sistema.

Dentre as áreas analisadas, o ponto 4 apresentou as melhores medidas bióticas. Localizado no início do represamento do Pitangui, este trecho parece estar menos impactado, apresentando uma mata ciliar melhor conservada e zonas de retenção e correnteza, condições que permitem a colonização de uma fauna aquática mais diversificada. A maior porcentagem de táxons fragmentadores (animais que utilizam folhas e outras partes de vegetais superiores alóctones), cuja abundância possivelmente esteja relacionada às áreas florestadas marginais, pode confirmar a melhor qualidade ambiental neste trecho.

Com relação às categorias tróficas, todos os pontos de amostragem apresentaram predomínio de táxons predadores, como os hirudíneos e os odonatos; e coletores e/ou predadores, como os Chironomidae, em sua maioria pertencentes ao gênero Chironomus (que totalizaram mais de $84 \%$ dos quironomídeos capturados). MARQues et al. (1999) também verificaram maior frequência de coletores e/ou predadores em duas lagoas do Parque Estadual do Rio Doce, em Minas Gerais e resultados semelhantes foram obtidos por CALLISTO et 
al. (2001) e PiEdRAs et al. (2006). A pequena porcentagem de fragmentadores, na maioria dos pontos investigados, pode estar relacionada a vários fatores. Segundo NISLOw \& Lowe (2006) existe uma relação negativa entre abundância deste grupo trófico e desmatamento e assoreamento de rios de cabeceira. Para MARQues et al. (1999), a quantidade e a qualidade inferior da matéria orgânica cedida pela vegetação ripária (alóctone) são alguns dos fatores responsáveis pela diminuição da abundância de organismos fragmentadores e raspadores. Os fragmentadores estão presentes em áreas com dossel denso, como em lagoas ou em rios com matas de galerias, onde coexistem com outras categorias, como os raspadores. Estes últimos podem ser mais comuns em áreas mais iluminadas, com maior disponibilidade de substratos. Os coletores e filtradores são mais frequentes em rios maiores e lagos, nos pontos de maior correnteza. Os predadores por serem menos restritivos, são encontrados em vários tipos de ambientes (VANNOTE et al., 1980; Williams \& FeltMATE, 1992).

Os valores de BMWP obtidos permitem caracterizar a qualidade das águas de Alagados como não poluída a levemente poluída, embora em vários pontos ao longo de sua extensão, a represa sofra diversos impactos como retirada de sua mata ripária e aporte de dejetos urbanos e animais. Por outro lado, de acordo com a classificação de Mandaville (2002), os valores de ASPT entre 4,0 e 5,0 dos cinco pontos amostrados revelam um grau de poluição moderado no sistema Alagados. Quando comparados os dois índices bióticos BMWP e ASPT, aparentemente, os valores de ASPT são os que melhor explicam a qualidade das águas da represa, tendo em vista que eles são mais coerentes com as demais medidas bioindicadoras discutidas anteriormente. $\mathrm{O}$ uso de índices bióticos em programas de monitoramento de bacias hidrográficas permite evidenciar de maneira mais abrangente as alterações nos corpos d'água que nem sempre são possíveis de serem detectadas através apenas de análises físico-químicas (SILVA et al., 2007; BAPTISTA, 2008; Monteiro et al., 2008; QueIROZ et al., 2008).

De maneira geral, os baixos índices de riqueza, diversidade, equitabilidade, porcentagem de táxons fragmentadores e do ASPT refletem certa deterioração das características ecológicas das águas de Alagados, decorrente de impactos relacionados a desestruturação de suas áreas marginais e contaminação do sistema por poluentes orgânicos, com consequente proliferação de cianobactérias.

Reforça-se, assim, a importância de mais estudos com macroinvertebrados aquáticos no biomonitoramento de ecossistemas da região e a criação de parâmetros regionais, para o acompanhamento dos efeitos antrópicos sobre a qualidade das águas de Alagados e como ferramenta para programas de recuperação e gestão integrada dos seus recursos.

Agradecimentos. Os autores agradecem ao PIBIC/CNPq/ UEPG, pela concessão das bolsas de iniciação científica; à SANEPAR - Ponta Grossa, pelo apoio nos trabalhos de campo e às biólogas Cristiane A. Lopes Costa e Eveline Zacalusne, pelo auxílio nas coletas e triagem do material. Agradecemos ainda a Fábio O. Roque pela leitura crítica do texto e sugestões.

\section{REFERÊNCIAS BIBLIOGRÁFICAS}

BAptista, D. F. 2008. Uso de macroinvertebrados em procedimentos de biomonitoramento em ecossistemas aquáticos. Oecologia Brasiliensis 12(3):425-441.

Bubinas, A. \& JagminienÉ, I. 2001. Bioindication of ecotoxicity according to community structure macrozoobenthic fauna. Acta Zoologica Lituanica 11(1):90-99.

Callisto, M.; Moreno, P. \& Barbosa, F. A. R. 2001. Habitat diversity and benthic functional trophic groups at Serra do Cipó, Southest Brazil. Revista Brasileira de Biologia 61(2):259-266.

Carvalho, A. L. \& Calil, E. R. 2000. Chaves de identificação para as famílias de Odonata (Insecta) ocorrentes no Brasil, adultos e larvas. Papéis Avulsos de Zoologia (São Paulo) 41(15):223-241

Cummins, K. W. \& Klug, M. J. 1979. Feeding ecology of stream invertebrates. Annual Review of Ecology and Systematics 10: $147-172$

Di Giovanni, M. V.; Goretti, E. \& Tamanti, V. 1996. Macrobenthos in Montedoglio Reservoir, central Italy. Hydrobiologia 321: $17-28$.

Dornfeld, C. B. \& Fonsech-Gessner, A. A. 2005. Fauna de Chironomidae (Diptera) associada à Salvia sp. e Myriophyllum sp. num reservatório do córrego do Espraiado, São Carlos, São Paulo, Brasil. Entomología y Vectores 12(2):181-192.

Fernández, H. R. \& Domínguez, E. 2001. Guía para la determinación de los artrópodos bentónicos sudamericanos. Tucumán, Universidad Nacional de Tucumán. 282p.

Fundação ABC (Pesquisa e Desenvolvimento Agropecuário). 2008. Sistema de monitramento agrometeorológico. Disponível em: <http://sma.fundacaoabc.org.br>. Acesso em: 13.10.2008.

Gonçalves, F. B. \& Aranha, J. M. R. 2004. Ocupação espaçotemporal pelos macroinvertebrados bentônicos na bacia do rio Ribeirão, Paranaguá, PR (Brasil). Acta Biológica Paranaense 33(1/2/3/4):181-191.

Heino, J. 2009. Biodiversity of aquatic insects: spatial gradients and environmental correlates of assemblage-level measures at large scales. Freshwater Reviews 2(1):1-29.

Jablonska, I. \& Paturej, E. 1999. The domination and constancy of occurrence of invertebrate communities in the Hancznska Bay, Lake Wigry, NW Poland. Acta Hydrobiologica 41(6):249-253.

Junqueira, V. M.; Amarante, M. C.; Dias, C. F. S. \& França, E. S. 2000. Biomonitoramento da qualidade das águas da bacia do Alto Rio das Velhas através de macroinvertebrados. Acta Limnologica Brasiliensia 12:73-87.

Junqueira, V. M. \& CAmpos, S. C. M. 1998. Adaptation of the "BMWP" method for water quality evaluation to Rio das Velhas watershed (Minas Gerais, Brazil). Acta Limnologica Brasiliensis 10(2): 125-135

Mandaville, S. M. 2002. Benthic macroinvertebrates in freshwaters: taxa tolerance values, metrics and protocols. Disponível em: <http://chebucto.ca/Science/SWCS/ SWCS.html>. Acesso em: 12.06.2009.

Margalef, F. 1983. Liminología. Barcelona, Omega. 1010p.

Marques, M. G. S. M.; Ferreira, R. L. \& Barbosa, F. A. R. 1999. A comunidade de macroinvertebrados aquáticos e características limnológicas das lagoas carioca e da barra, Parque Estadual do Rio Doce, MG. Revista Brasileira de Biologia 59(2):203-210.

McCafferty, W. P. 1981. Aquatic entomology. Boston, Jones and Bartlett. 448p.

Melo, A. S. \& Hepp, L. U. 2008. Ferramentas estatísticas para análise de dados provenientes de biomonitoramento. Oecologia Brasiliensis 12(3):463-486.

Merritt, R. W. \& Cummins, K. W. 1996. An introduction to the aquatic insects of North America. 3. ed. Dubuque, Kendal/ Hunt. 862p.

MMA (Ministério do Meio Ambiente). 2005. Resolução no 357, de 17 de março de 2005. Disponível em: <http://www.mma.gov.br/ port/conama/res/res05/res35705.pdf>. Acesso em: 02.04.2009.

Monteiro, T. R.; Oliveira, L. G. \& Godoy, B. S. 2008. Biomonitoramento da qualidade de água utilizando macroinvertebrados bentônicos: adaptação do índice biótico 
BMWP à bacia do rio Meia Ponte-GO. Oecologia Brasiliensis 12(3):553-563.

Moro, J. C.; Costa, E. T. V.; Milanese, S. \& Moro, R. S. 2005. Comparação da cobertura vegetal nas áreas de preservação permanente na represa de Alagados (PR), de 1980 a 2001. Publicatio UEPG Ciências Biológicas e da Saúde 11(2): 13-20.

Moro, R. S.; Ferrari, F.; Santos, M. A.; Barros, K. F. \& Schimitt, J. 2003. Heterogeneidade espacial do fitoplâncton da represa de Alagados (Ponta Grossa, PR). Publicatio UEPG Ciências Biológicas e da Saúde 1(1):21-30.

Nieser, N. \& Melo, A. L. 1997. Os heterópteros aquáticos de Minas Gerais. Belo Horizonte, EDUFMG. 177p.

Nislow, K. H. \& Lowe, W. H. 2006. Influences of logging history and riparian forest characteristics on macroinvertebrates and brook trout (Salvelinus fontinalis) in hedwater streams (New Hampshie, U.S.A.). Freshwater Biology 51:388-397.

Oliveira, A.; Morgan, F. L.; Moreno, P. \& Callisto, M. 2005. Inventário da fauna de insetos aquáticos na estação Ambiental de Peti (CEMIG). In: Silveira, F. ed. Anais da ANEEL projeto Peti/UFMG. Belo Horizonte, EDUFMG. p.25-30.

Pamplin, P. A. Z.; Almeida, T. C. M. \& Rocha, O. 2006. Composition and distribution of bentic macroinvertebrates in Americana Reservoir, SP, Brazil. Acta Limnologica Brasiliensia 18(2):121-132.

Pamplin, P. A. Z. \& Rocha, O. 2007. Temporal and bathymetric distribution of benthic macroinvertebrates in the Ponte Nova Reservoir, Tietê river (São Paulo, Brazil). Acta Limnologica Brasiliensia 19:439-452.

Pamplin, P. A. Z.; Rocha, O. \& Marchese, M. 2005. Riqueza de espécies de Oligochaeta (Annelida, Clitellata) em duas represas do rio Tietê (São Paulo). Biota Neotropica 5(1):63-70.

Peiró, D. F. \& Alves, R. G. 2006. Insetos aquáticos associados a macrófitas da região litoral da represa do ribeirão das Anhumas (município de Américo Brasiliense, São Paulo, Brasil). Biota Neotropica 6:1-9.

Piedras, S. R. N.; Bager, A.; Moraes, P. R. R.; Isoldi, L. A.; Ferreira, O. G. L. \& Heemann, C. 2006. Macroinvertebrados bentônicos como indicadores de qualidade de água na barragem Santa Bárbara, Pelotas, RS, Brasil. Ciência Rural 36(2):494-500.

Pinto-Coelho, R. M.; Coelho, M. M.; Espirito-Santo, M. M. \& Cornelissen, T. G. 1999. Efeitos da eutrofização na comunidade planctônica da lagoa da Pampulha, Belo Horizonte, MG. In: HENRY, R. ed. Ecologia de reservatórios: estrutura, função e aspectos sociais. Botucatu, FUNDBIO/FAPESP. p.551-572.

Queiroz, J. F.; Ferraz, J. M. C.; Silveira, M. P.; SitTon, M.; Marigo, A. L. S.; Carvalho, M. P. \& Ribacinko, D. B. 2008. Avaliação preliminar da qualidade da água em duas microbacias do rio Mogi (SP). Circular Técnica - Embrapa Meio Ambiente, n.17. 11p.
Queiroz, J. F.; Trivinho-Strixino, S. \& Nascimento, V. M. C. 2000. Organismos bentônicos bioindicadores da qualidade das águas da Bacia do Médio São Francisco. Comunicado Técnico - Embrapa Meio Ambiente, n.3. 4p.

Scheffer, E. W. O. \& Busch, O. M. S. 2010. Qualidade da água. In Gealh, A. M.; Melo, M. S. \& Moro, R. S. eds. Pitangui, rio de contrastes: seus peixes, seus lugares e sua gente. Ponta Grossa, UEPG. p.43-51.

Sepka, E. R.; Barbola, I. F.; Moraes, M. F. P. G.; Zacalusne, E. \& Nascimento, E. A. 2005. Invertebrados aquáticos como bioindicadores da qualidade da água da represa de Alagados, em Ponta Grossa, PR. In: SIMPÓSIO SUL DE GESTÃO E CONSERVAÇÃO AMBIENTAL, $1^{\circ}$, Erechim, 2005, Anais... Erechim, Toppa, R. H. \& Hepp, H. U. p.259-267.

Silva, F. L.; Moreira, D. C.; Bochini, G. L. \& Ruiz, S. S. 2007. Desempenho de dois índices biológicos na avaliação da qualidade das águas do córrego Vargem Limpa, Bauru, São Paulo, através de macroinvertebrados bentônicos. Pan-American Journal of Aquatic Sciences 2(3):231-234.

SouzA, A. H. F. F. \& Abílio, F. J. P. 2006. Zoobentos de duas lagoas intermitentes da caatinga paraibana e as influências do ciclo hidrológico. Revista de Biologia e Ciências da Terra, Suplemento Especial (1):146-164.

Stenert, C.; Santos, E. M. \& Maltchik, L. 2004. Levantamento da diversidade de macroinvertebrados em áreas úmidas do Rio Grande do Sul (Brasil). Acta Biologica Leopoldensia 26(2):225-240.

TARDivo, R. C.; BACH, A. \& Moro, R. S. 2007. Macrófitas aquáticas da represa de Alagados, vegetação aquática. In: Melo, M. S.; Moro, R. S. \& Guimarães, G. B. eds. Patrimônio Natural dos Campos Gerais do Paraná. 1. ed. Ponta Grossa, UEPG. p. $105-108$

Trivinho-Strixino, S. \& Strixino, G. B. M. A. 1995. Larvas de Chironomidae (Diptera) do estado de São Paulo: guia de identificação e diagnose dos gêneros. São Carlos, PPG-ERN/UFSCar. 229p.

Valenti, W. C. \& Froehlich, O. 1986. Estudo da diversidade da taxocenose de Chironomidae de dez reservatórios do Estado de São Paulo. Ciência e Cultura 38(4):703-707.

Vannote, R. L.; Minshall, G. W.; Cummins, K. W.; Sedell, J. R. \& Cushing, C. E. 1980. The river continuum concept. Canadian Journal of Fisheries and Aquatic Sciences 37:130-137.

Wilhm, J. \& Dorris, T. 1968. Biological parameters for water quality criteria. Biological Science 18:477-381.

Williams, D. D. \& Feltmate, B. W. 1992. Aquatic Insects. Trowbridge, Redwood Books. 358p.

Zinchenko, T. D. 1992. Long term (30 days) dynamics of Chironomidae (Diptera) fauna in the Kuibyshev water reservoir associated with eutrophication processes. Netherlands Journal of Aquatic Ecology 26(2/4):533-542. 